\title{
Multi-leptons and top-jets in the hunt for gluinos in R-parity violating supersymmetry
}

\author{
Sanjoy Biswas, ${ }^{a}$ Diptimoy Ghosh $^{b}$ and Saurabh Niyogi ${ }^{c}$ \\ a Dipartimento di Fisica, Universitá di Roma "La Sapienza", \\ Piazzale A. Moro 2, I-00185 Roma, Italy \\ ${ }^{b}$ INFN - Sezione di Roma, \\ Piazzale A. Moro 2, I-00185 Roma, Italy \\ ${ }^{c}$ Regional Centre for Accelerator-based Particle Physics, Harish-Chandra Research Institute, \\ Chhatnag Road, Jhusi, Allahabad - 211 019, India \\ E-mail: sanjoy.biswas@roma1.infn.it, diptimoy.ghosh@roma1.infn.it, \\ sourabh@hri.res.in
}

ABSTRACT: The presence of R-parity $\left(\mathcal{R}_{p}\right)$ violation offers intersting decay channels for the gluinos. In this work we present a new search strategy for the gluinos in the presence of semileptonic $\mathcal{R}_{p}$ violating couplings $\lambda_{133}^{\prime}$ and $\lambda_{233}^{\prime}$. We consider two scenarios (i) $\lambda^{\prime}$ induced 3-body decay of gluinos to a top quark $(t)$, a bottom quark $(b)$ and a light lepton $(\ell)$ (ii) cascade decay of gluinos to top quarks and neutralinos $\left(\widetilde{\chi}_{1}^{0}\right)$ followed by the decay of $\widetilde{\chi}_{1}^{0}$ to $t, b$ and $\ell$ through $\lambda^{\prime}$ couplings. We present two different search procedures which are common to both the scenarios. While the first one involves the traditional approach with multi-leptons and $b$-tagged jets, the second one employs the more recent technique to reconstruct highly energetic hadronically decaying top quarks. We perform a detailed simulation of the signal as well as all the relevant Standard Model backgrounds to show that the second procedure offers slightly better sensitivity for gluino discovery. In both the procedures, $\mathrm{a} \geq 5 \sigma$ discovery is possible for the gluino mass in the range $1.5-1.7 \mathrm{TeV}$ at $14 \mathrm{TeV}$ LHC with $50 \mathrm{fb}^{-1}$ integrated luminosity.

KEYwORDS: Supersymmetry Phenomenology

ARXIV EPRINT: 1312.0549 


\section{Contents}

1 Introduction 1

2 RPV SUSY 3

3 Signal, backgrounds and our search strategy 4

3.1 Multilepton signal 5

3.2 Boosted top 6

4 Results and discussion $\quad 8$

4.1 Multi-lepton signal 8

$\begin{array}{lll}4.2 & \text { Boosted top } & 10\end{array}$

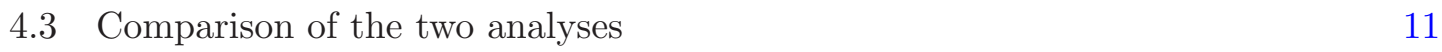

5 Conclusion $\quad 12$

\section{Introduction}

The discovery of the Standard Model (SM) higgs-like particle [1, 2] in the successful 8-TeV run of the Large Hadron Collider (LHC) has left us in a situation, as never before, where any significant excess of signal would now definitely point towards new physics (NP) beyond the SM. The Minimal Supersymmetric Standard Model (MSSM) [3-6], being one of the most attractive possibilities beyond the SM, would be the prime candidate to be searched for in various possible channels at the $14-\mathrm{TeV}$ LHC. While Supersymmetry (SUSY) is clearly a broken symmetry, the demand of naturalness of the electroweak scale strongly suggests that at least some of the SUSY partners of the SM particles appear around a (few) TeV scale. The large amount of data collected at the 7 and $8-\mathrm{TeV}$ run of the LHC, however, has already pushed the lower bounds of many of the SUSY partners well above a TeV. For example, the bound on the mass of the gluinos and the first two generation of squarks stand roughly around $1.5 \mathrm{TeV}$ in the constrained version of the R-parity $\left(\mathcal{R}_{p}\right)$ conserving MSSM $[7,8]$. Indirect bounds from the measured value of the higgs boson mass and the low energy flavour observables also stand in the same ballpark [9-13]. ${ }^{1}$

While in one hand this has made many theorists suspicious about the idea of naturalness, on the other hand this has also motivated many others to question the assumptions underlying the LHC searches, e.g., the assumption of $\mathcal{R}_{p}$ conservation. Note that the conservation of $\mathcal{R}_{p}$ renders the lightest SUSY particle (LSP) stable which often leads to large

\footnotetext{
${ }^{1}$ However, in general MSSM scenarios the bounds from higgs mass and flavour observables are much weaker.
} 
missing energy $\left(\mathrm{E}_{\mathrm{T}}\right)$ in the detectors. As large $\mathrm{E}_{\mathrm{T}}$ has been widely used by the experimental searches to suppress backgrounds, the $\mathcal{R}_{p}$ violating scenarios not only help evade the existing experimental constraints, but also open up a plethora of new possibilities and rich collider phenomenology.

Note that the lower bound on the mass of the top squark (stop) is rather model dependent even for the $\mathcal{R}_{p}$ conserving case; its mass around a $\mathrm{TeV}$ (or a few hundred $\mathrm{GeV}$ less) is perfectly allowed in spite of the wealth of LHC data $[14,15]$. A large number of phenomenological studies has been devoted to stop searches both in the $\mathcal{R}_{p}$ conserving [16$24]$ as well as $\mathcal{R}_{p}$ violating [25-28] scenarios. On the other hand, the bound on the gluino mass, in general, is stronger and the current lower value is more than a TeV even in most of the simplified scenarios (if the neutralino is not too heavy) $[8,29]$. Hence, the $\mathcal{R}_{p}$ violating MSSM (thus, a very low MET) is an attractive possibility to realize $\mathrm{TeV}$ scale gluinos. This has motivated a number a studies focusing on signatures of gluino pair production in specific $\mathcal{R}_{p}$ violating scenarios [27, 30-35]. For example, in reference [33] the authors considered the baryonic $\mathcal{R}_{p}$ violating couplings and obtained a lower bound of $800 \mathrm{GeV}$ on the gluino mass using the CMS data in the same sign di-leption $+\mathrm{E}_{\mathrm{T}}+b$-jet channel. These authors also reported an expected bound of $1.45 \mathrm{TeV}$ on the gluino mass in their simplified scenario from the $14 \mathrm{TeV}$ LHC with $100 \mathrm{fb}^{-1}$ integrated luminosity. On the experimental front also many of the $\mathcal{R}_{p}$ violating scenarios are now being challenged; both the ATLAS and CMS collaborations have reported bounds on the masses of some MSSM particles in several $\mathcal{R}_{p}$ violating simplified scenarios [36-40].

In this work, we consider the presence of semileptonic $\mathcal{R}_{p}$ violating operators in the context of the gluino searches. We reiterate that the bound on the gluino mass is less model dependent (apart from the requirement of large MET) than the bound on stop mass as the production and decay of the gluinos mainly involve the QCD part of the MSSM lagrangian. However, in the decay cascade other parameters also come into play bringing in additional model dependence. In view of this, here we consider two simplified models consistent with the latest bounds. In the first case we assume that the top squark is heavier than the gluino $\left(m_{\widetilde{g}}<m_{\widetilde{t}_{1}}\right)$ so that the $\widetilde{g}$ decays through $\tilde{g} \rightarrow t b \ell$ via an off-shell top squark $\left(\widetilde{t}_{1}\right)$ (see section 2 and 3 for details). In this case the top squark is required to be left-handed in order that it decays through the $\lambda^{\prime}$ coupling (see next section). Note that in this scenario the heavy-ness of the stop evades the possible bound from the $b-\ell$ resonance searches at the LHC [41-45]. In the second scenario we assume the opposite hierarchy $m_{\widetilde{g}}>m_{\widetilde{t}_{1}}$ so that the gluinos decay though $\tilde{g} \rightarrow \widetilde{t}_{1}\left(\rightarrow t \tilde{\chi}_{1}^{0}\right) t$. The $\tilde{\chi}_{1}^{0}$ now decays through $\tilde{\chi}_{1}^{0} \rightarrow t b \ell$ in the presence of the same $\lambda^{\prime}$ coupling.

In both the cases the final state has top quarks, multiple leptons and $b$-jets. At first we investigate the traditional tri-leptons $+2 b$-jets final state. Note that we have chosen this final state in order that the same analysis can be applied to both the cases mentioned above. Proceeding further, we then point out that owing to the high mass of the gluinos some of the top quarks in the final state would often carry rather large transverse momentum and would give rise to collimated jets. We find that the use of jet-substructure techniques to tag these energetic "top-jets" are indeed very powerful to discover a signal.

The paper is organized as follows. In the next section we briefly discuss the simplified $\mathcal{R}_{p}$ violating scenario that we consider in our study. The details of signal and the back- 
grounds along with our search strategy is discussed in section 3. In section 4 we present the summary of our simulation and discuss the results. Finally, we conclude in section 5.

\section{RPV SUSY}

R-parity is a discrete symmetry of the MSSM lagrangian defined such a way that all the SM particles have $\mathcal{R}$-charge +1 and all their superpartners have $\mathcal{R}$-charge -1 . The above charge assignment allows $\mathcal{R}_{p}$ to be related to the Baryon $(B)$ and Lepton $(L)$ numbers by the following simple formula,

$$
\mathcal{R}_{p}=(-1)^{2 S}(-1)^{3(B-L)},
$$

where $S$ is the spin of the particle. The above formula makes it explicit that the conservation of $\mathcal{R}_{p}$ forbids all the dimension- 4 and 5 proton decay operators. As all the SUSY particles are odd under $\mathcal{R}_{p}$, this also makes the LSP stable providing a good dark matter candidate.

However, it is possible that $\mathcal{R}_{p}$ is violated in specific ways that do not introduce fatal rates for proton decay. For example, switching on either $B$ or $L$ violating $\mathcal{R}_{p}$ violating couplings but not the both still forbids the dangerous proton decay operators.

In the absence of $\mathcal{R}_{p}$ the additional marginal and relevant terms allowed by gauge invariance in the MSSM superpotential can be written as [46-48],

$$
W_{\mathcal{R}_{p}} \supset \frac{1}{2} \lambda_{i j k} L_{i} L_{j} E_{k}^{c}+\lambda_{i j k}^{\prime} L_{i} Q_{j} D_{k}^{c}+\frac{1}{2} \lambda_{i j k}^{\prime \prime} U_{i}^{c} D_{j}^{c} D_{k}^{c}+\mu_{i} L_{i} H_{u}
$$

where $L_{i}\left(E_{i}^{c}\right)$ are left-handed lepton doublet (right-handed lepton) superfields, $Q_{i}\left(U_{i}^{c}, D_{i}^{c}\right)$ the left-handed quark doublet superfields (right-handed Up-type and Down-type quark superfields, respectively) and $H_{u}$ is the Higgs superfield that gives mass to the up-type quarks.

Clearly, the couplings $\lambda^{\prime \prime}$ violate $B$, while the couplings $\lambda$ and $\lambda^{\prime}$ violate $L$. As both $B$ and $L$ number must be violated to induce proton decay, it is still possible to turn on either $\lambda^{\prime}$ or $\lambda^{\prime \prime}$ coupling without spoiling proton stability. Certain combinations of these $\mathcal{R}_{p}$ violating couplings are also constrained from various low energy observables (such as FCNC decays, neutron-antineutron oscillation, neutrino oscillation data, neutrino-less double beta decay, decays of tau lepton, meson mixing etc.) as well as data from high energy colliders e.g., LEP and Tevatron, see e.g., [49] and the references therein.

In this work we consider the presence of $\lambda^{\prime}$ couplings in particular, $\lambda_{133}^{\prime}$ and $\lambda_{233}^{\prime}$, giving rise to the possibility of stop (coming from gluino decays in our case) decaying to a $b$-quark and light leptons (electron for $\lambda_{133}^{\prime}$ and muon for $\lambda_{233}^{\prime}$ ). Note that the $\lambda^{\prime}$-type couplings are also constrained to some extent by the various measurements mentioned above. However, the constraints on the couplings $\lambda_{133}^{\prime}$ and $\lambda_{233}^{\prime}$ are less severe as these involve the third generation quarks. The strongest bounds arise from the Majorana neutrino mass $[49,50]$ and flavor violating top decays [51]. The $Z$-partial width also constrains these couplings but to a lesser extent than the previous ones [52]. However, these bounds are not strong enough to make the gluinos and neutralinos stable in the detectors. 


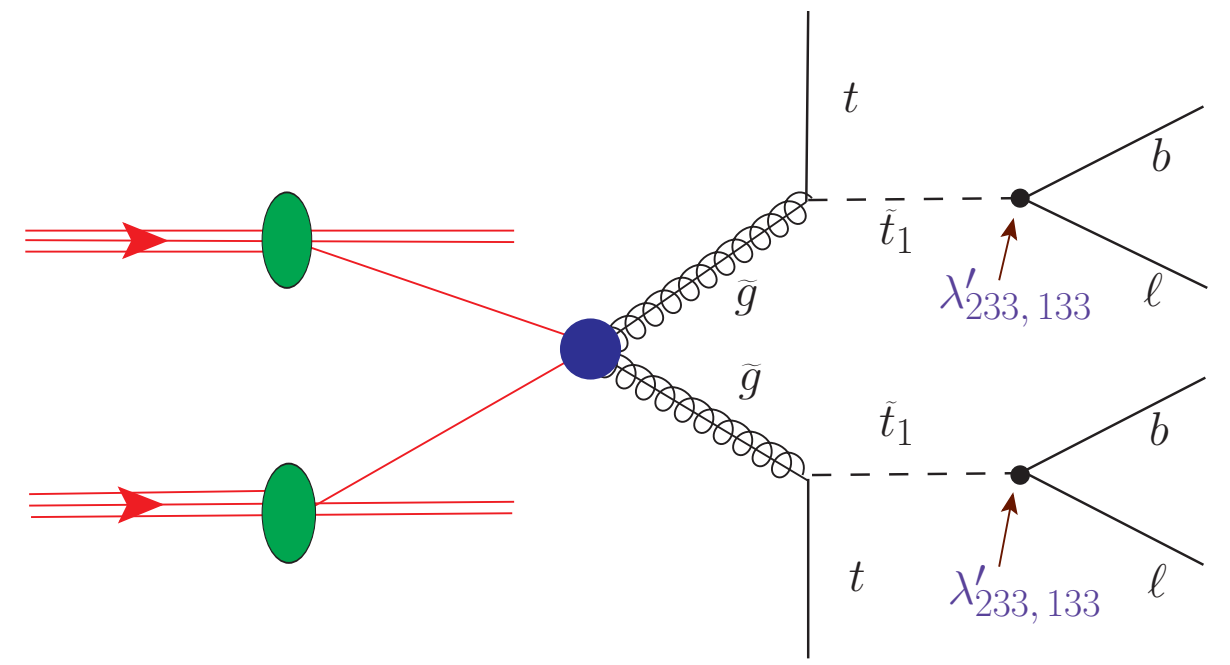

Figure 1. The relevant Feynman diagram for gluino production followed by its decay to $(t b \ell)$ final state in the presence of $\lambda^{\prime}$ couplings in our $\mathcal{R}_{p}$ violating scenario-1.

Although, ideally one should explain why some of these couplings are extremely small and some other are not, this is certainly quite interesting phenomenologically as it opens up many new decay channels of the MSSM particles leading to a rich phenomenology which should be studied in the colliders. As mentioned in the introduction, because of the absence of sufficient $\mathrm{E}_{\mathrm{T}}$ this also lowers the bounds on some of the SUSY particles.

In the next section we will now discuss the specific decay topologies which are considered in this work along with the details of our simulation procedures.

\section{Signal, backgrounds and our search strategy}

As mentioned in the introduction, in this work we consider gluino pair production followed by two different decay chains. In the first case, the $\widetilde{g}$ decays to $(t b \ell)$ through an offshell stop:

$$
p p \rightarrow \widetilde{g} \widetilde{g}, \quad \widetilde{g} \rightarrow t b \ell \quad(\text { scenario }-1) .
$$

A sample Feynman diagram is shown in figure 1. Looking at the structure of the $\lambda^{\prime}$ coupling (eq. (2.2)) reveals that the top squark in this case has to have large left-handed component.

In the second case we assume the decay chain,

$$
p p \rightarrow \widetilde{g} \widetilde{g}, \quad \widetilde{g} \rightarrow \widetilde{t}_{1} t, \widetilde{t}_{1} \rightarrow t \widetilde{\chi}_{1}^{0}, \quad \widetilde{\chi}_{1}^{0} \rightarrow t b \ell \quad(\text { scenario }-2) .
$$

Figure 2 shows a sample Feynman diagram for this process where the $\widetilde{\chi}_{1}^{0}$ is assumed to decay through the left-handed component of an off-shell stop. ${ }^{2}$

\footnotetext{
${ }^{2}$ The same decay $\tilde{\chi}_{1}^{0} \rightarrow t b \ell$ can also proceed via an off-shell left-handed slepton. However, in that case the branching ratio can not be too high (typically less than 0.5 ) because of the presence of the left-handed sneutrino in the spectrum with the same mass (as the left-handed charged slepton), thus giving rise to also the decay $\widetilde{\chi}_{1}^{0} \rightarrow b \bar{b} \nu$. In fact, exactly these two decay chains (starting from stop pair production) were considered by the CMS collaboration to obtain a bound of about $700 \mathrm{GeV}$ on the stop mass if $\widetilde{\chi}_{1}^{0}$ is not too heavy [36].
} 


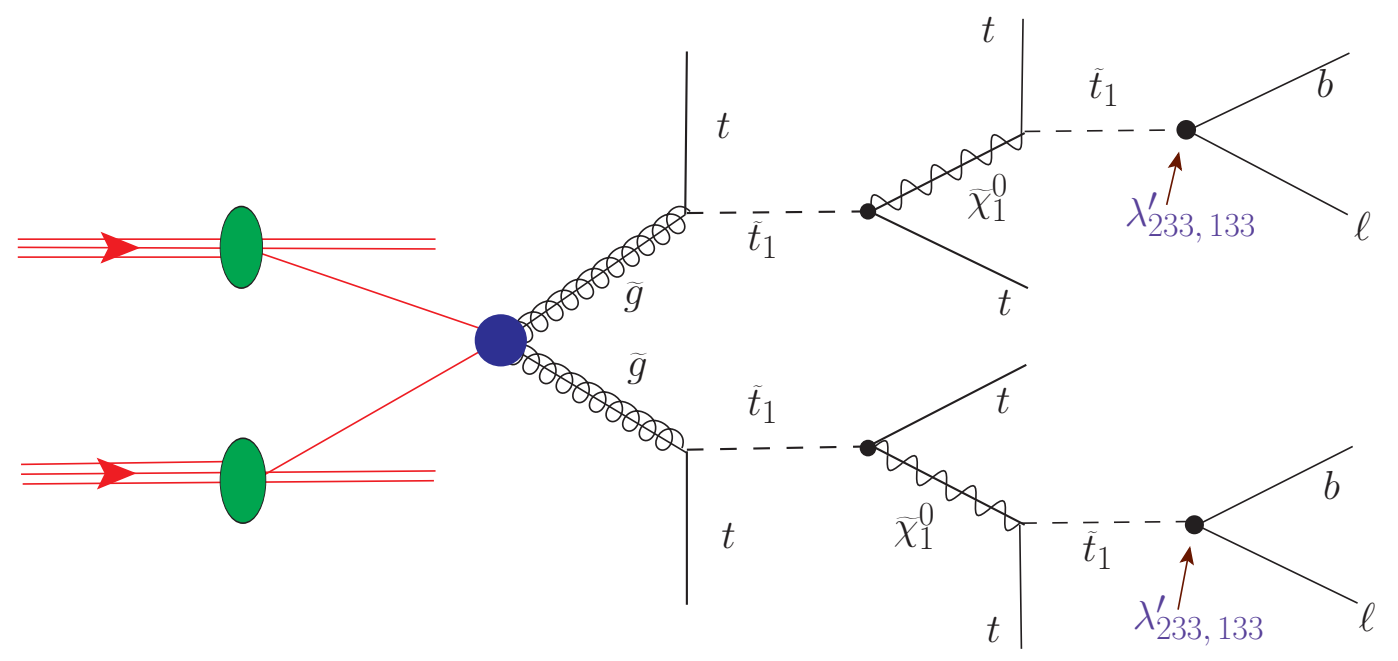

Figure 2. The relevant Feynman diagram for gluino production followed by it's cascade decay to $t, b$ and leptons through $\lambda^{\prime}$ couplings in our $\mathcal{R}_{p}$ violating scenario-2.

In the following section we describe the details of our simulation procedure as well as the kinematic selection cuts for the signal and the relevant backgrounds. For simplicity, we will assume all the relevant branching ratios to be unity while presenting the results. For other values of the branching ratios our results can be easily scaled down appropriately.

\subsection{Multilepton signal}

We are now in a position to discuss the details of our event selection procedure. In this section we describe our analysis with multi-leptons and $b$-tagged jets. The analysis involving the tagging of top-jets will be discussed in the next subsection.

In the multi-lepton analysis, we first reconstruct the jets using the simple cone algorithm with the value of the radius parameter $R=0.4$. We consider only those jets which satisfy a transverse momentum cut $p_{T}^{j} \geq 20 \mathrm{GeV}$ and the pseudo-rapidity $|\eta| \leq 2.5$. Leptons are also selected with a transverse momentum $p_{T}^{\ell} \geq 10 \mathrm{GeV}$ and the pseudo-rapidity $|\eta| \leq 2.5$. We call a lepton isolated if it satisfies

(i) The distance between the lepton and any of the jets $\Delta R(j \ell)>0.4$

(ii) The distance between the lepton and any of the other leptons $\Delta R(\ell \ell)>0.2$

(iii) The ratio of the total hadronic transverse energy deposit within a cone of $\Delta R=0.2$ around the lepton to the lepton transverse energy is $\leq 0.15$.

A jet is identified as a $b$-jet if it is close $(\Delta R<0.2)$ to a $b$-quark. For the $b$-tagging efficiency $\left(\epsilon_{b}\right)$ we use the prescription from reference [53] which gives $\epsilon_{b}=0.71$ for $90<$ $p_{T}<170 \mathrm{GeV}$ and at higher (lower) $p_{T}$ it decreases linearly with a slope of $-0.0004(-0.0047)$ $\mathrm{GeV}^{-1}$. Moreover, the probability of mis-tagging a $c$-jet (light jet) as a $b$-jet is taken to be $20 \%(0.73 \%)$ [54]. Once the leptons and jets are constructed we use further analysis cuts for selecting events. Since the decay topologies in the two scenarios considered here 
are quite different, $p_{T}$ distributions of the observed leptons are expected to be different as well. In scenario-1, leptons are coming directly from the decay of a heavy gluino. Hence they are much more likely to pass harder $p_{T}$ cuts. On the other hand, leptons coming at a much later stage in the decay chain in scenario-2, are expected to be less energetic on the average. Therefore, it seems legitimate to employ different sets of lepton $p_{T}$ cuts for the two scenarios. We also reject (veto) an event if it has two opposite-charge-same-flavor leptons with invariant mass around the $Z$-boson mass.

We use the following set of selection criteria in scenario-1:

- Cut-I: we demand that the event contains at least 3 isolated leptons. The three leptons must satisfy $p_{T_{\ell_{1}}}>50 \mathrm{GeV}, p_{T_{\ell_{2}}}>40 \mathrm{GeV}$ and $p_{T_{\ell_{3}}}>30 \mathrm{GeV}$. In addition, we also require that the event has at least 2 jets.

- Cut-II: veto on $Z$-boson. We choose the mass window to be $M_{Z} \pm 10 \mathrm{GeV}$.

- Cut-III: the event must have at least two $b$-tagged jets.

- Cut-IV: we define the effective mass of an event to be $M_{\mathrm{eff}}=\sum_{j} p_{T}^{j}+\sum_{\ell} p_{T}^{\ell}+\mathbb{E}_{\mathrm{T}}$ and demand that the event satisfies $M_{\text {eff }}>1000 \mathrm{GeV}$.

In scenario-2 we slightly change the $p_{T}$ cuts on the final state leptons. We demand the 3 leptons satisfy the $p_{T}$ cuts of 40,30 and $20 \mathrm{GeV}$ respectively instead of 50,40 and $30 \mathrm{GeV}$ as used in the previous case.

For the simulation of signal events we have used Pythia-6.4.24 [55]. The Standard Model backgrounds have been generated using Alpgen v2.13 [56] with the MLM prescription [57] for the matching of matrix element hard partons and shower generated jets. We have used the CTEQ6L [58] parton distribution function for our simulations.

\subsection{Boosted top}

In this subsection we describe our second search strategy which involves tagging an energetic top qurak using the jet substructure technique. We use the Johns Hopkins top tagger (JHTopTgger) [59] in this work. We now briefly discuss the steps of the JHTopTgger algorithm mentioning our choice of specific parameters as and when the occasion arises. ${ }^{3}$ Here we closely follow the discussion in [59].

1. In the first step of the algorithm all the hadronic final states are clustered into the so-called 'fat-jets' using the Cambridge-Aachen (CA) algorithm [62] with the angular distance parameter $R=1.0$. In the $\mathrm{CA}$ algorithm one starts with all the four-momenta of the hadronic final states and then combine the pair which has the smallest $\Delta R \equiv \sqrt{\Delta \eta^{2}+\Delta \phi^{2}}$ (and $\Delta R<R$ ). The process is continued until there are no four-momenta left with $\Delta R<R$. The list of four-momenta which survive at this stage are then identified as jets.

\footnotetext{
${ }^{3}$ We have used the public package FastJet $[60,61]$ where this algorithm has been implemented.
} 
2. In the second step of the algorithm one fat-jet (say, $J$ ) is considered at a time and it's 4 -momenta $p^{(J)}$ is declustered into the 4-momenta $\left(p^{(j 1)}\right.$ and $\left.p^{(j 2)}\right)$ of the two subjets ( $j 1$ and $j 2$ ) which were combined to get the fat-jet $J$. As the clustering history is stored at each stage of the CA algorithm, this step is same as reversing the clustering process mentioned in the previous paragaraph.

3. Three quantities are now computed,

$$
\delta_{1}=p_{T}^{(j 1)} / p_{T}^{(J)}, \delta_{2}=p_{T}^{(j 2)} / p_{T}^{(J)} \text { and } \delta=\left|\eta^{j 1}-\eta^{j 2}\right|+\left|\phi^{j 1}-\phi^{j 2}\right| .
$$

The fat-jet $J$ is considered irreducible and having no substructure if the algorithm encounters at least one of the following two situations,

- $\delta_{1}, \delta_{2}<\delta_{p}$

- $\delta<\delta_{r}$

Here $\delta_{p}$ and $\delta_{r}$ are two adjustable parameters of the algorithm. In our analysis we set their values to $\delta_{p}=0.1$ and $\delta_{r}=0.2$.

4. If one of $\delta_{1}$ or $\delta_{2}$ comes out to be less than $\delta_{p}$, the corresponding subjet is discarded and the declustering procedure is applied to the other harder subjet. If both $\delta_{1}$ or $\delta_{2}$ are greater than $\delta_{p}$ then the declustering procedure is applied on both of them. This procedure stops when one of the conditions mentioned in step-3 is encountered or there is only one calorimeter cell left for the jet to be declustered.

5. The fat-jets with 3 or 4 subjets are kept for further analysis. The following three additional kinematic criteria are imposed before calling a fat-jet top tagged,

- The 3 or 4 subjets should reconstruct near the top quark mass. We use the top quark mass window to be $m_{t} \pm 20 \mathrm{GeV}$.

- One pair of subjets (one of the combinations $(1,2),(1,3),(1,4),(2,3),(2,4)$, $(3,4))$ should reconstruct near the $W$ mass. The $W$ mass window is chosen to be (60-100) $\mathrm{GeV}$ in our analysis.

- The $W$ helicity angle $\theta_{h}$ satisfies $\cos \theta_{h}<0.7$. The helicity angle is defined as the angle between the 3-momentum of the reconstructed top quark and that of one of the the $W$ boson's decay products (the lower $p_{T}$ subjet in this case, as was proposed in the original work [59] also), as measured in the rest frame of the reconstructed $W$.

Once the fat-jet satisfies the above kinematic cuts it is considered to be a true top candidate. Note that the subjet (or the hardest of the two subjets in case of four subjets) which is left after the $W$ reconstruction (we call this subjet as the non- $W$ subjet) is expected to be a $b$-jet for a true top candidate. In this work however, we do not demand that the non- $W$ subjet be $b$-tagged. In figure 3 we show the reconstruction of top quark mass for our signal benchmark points (see table 1). 

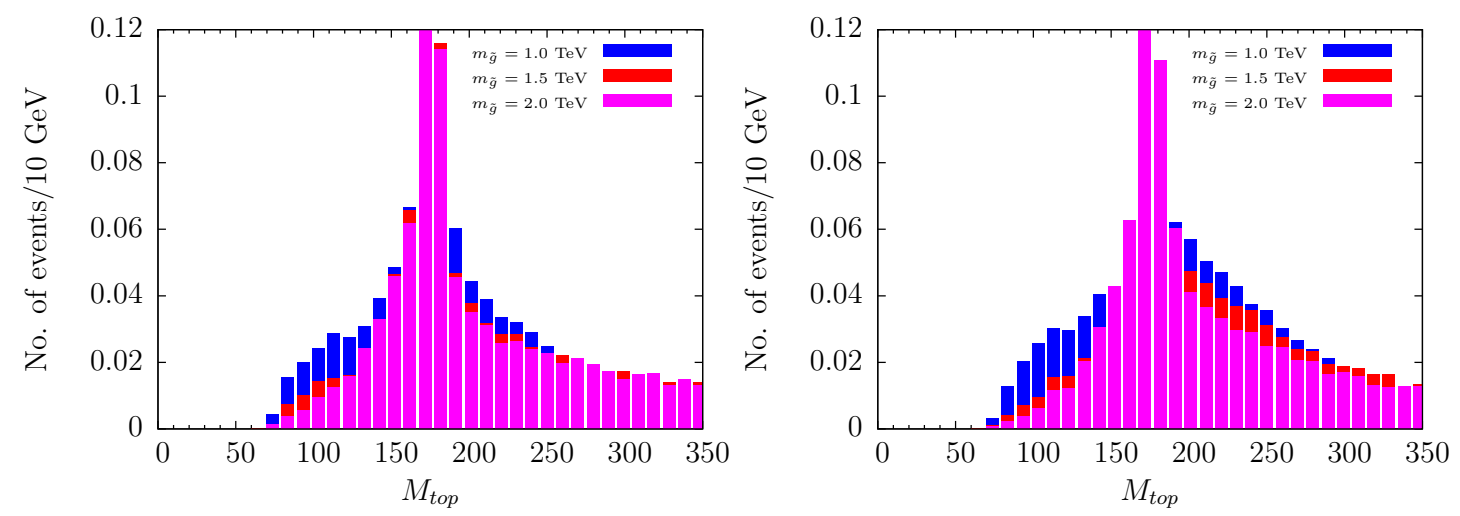

Figure 3. The distribution of the mass of the reconstructed top quark from the hardest fat-jet in our $\mathcal{R}_{p}$ violating scenario-1 (left panel) and scenario-2(right panel) for the benchmark points chosen in table 1 and table 2 respectively. The total number of events has been normalized to unity.

Once we find a top quark in an event (Cut-I) we then apply a few more selection criteria in order to combat the backgrounds. We discuss them below one by one.

Cut-II We demand at least two isolated leptons $(\ell 1$ and $\ell 2)$ with $p_{T}>50 \mathrm{GeV}$. We use the same isolation criteria used in the previous section 3.1.

Cut-III Apart from the $b$-jets coming from the top decays, the signal also has additional $b$-jets coming from gluino or neutralino decays. Keeping this mind we demand 2 $b$-tagged jets $(b 1$ and $b 2)$ which are far $(\Delta R>0.8)$ from the non- $W$ subjet of the reconstructed top quark. If an event has more than one reconstructed top quarks then only the hardest of them is used. The $b$-tagging procedure is again identical to that used in the previous subsection.

Cut-IV As a final selection criterion we demand that the effective mass of an event satisfies $M_{\mathrm{eff}}>1250 \mathrm{GeV}$. The definition of $M_{\mathrm{eff}}$ is identical to that used in the previous section.

\section{Results and discussion}

\subsection{Multi-lepton signal}

In table 1 and 2, we present the result of our analysis with $3 \ell+2 b+$ jets (section 3.1 ) for scenarios 1 and 2 respectively. The first three columns show the processes studied, the raw production cross-section and the number of events generated for the signal and background processes. The raw production cross-sections for the signal points correspond to the next-to-leading-order value calculated using Prospino [63] with default choices for the scale and the parton distribution function. For the background processes we use either the NLO cross-sections if they are available in the literature or the cross-sections obtained from Alpgen. For both the signal as well as the backgrounds the total number of events simulated is of the same order or more than the number expected in the $14 \mathrm{TeV}$ LHC with $50 \mathrm{fb}^{-1}$ luminosity (except for $t \bar{t}+$ jets). In the columns $4-7$ the number of events after 


\begin{tabular}{|c|c|c|c|c|c|c|c|c|}
\hline \multirow[b]{2}{*}{ Process } & \multirow{2}{*}{\multicolumn{2}{|c|}{$\begin{array}{l}\text { Production } \\
\text { cross-section }\end{array}$}} & \multirow[b]{2}{*}{$\begin{array}{c}\text { Simulated } \\
\text { events }\end{array}$} & \multicolumn{4}{|c|}{ No. of events after the cut } & \multirow[b]{2}{*}{$\begin{array}{l}\text { Final cross- } \\
\text { section (fb) }\end{array}$} \\
\hline & & & & $\mathrm{C} 1$ & $\mathrm{C} 2$ & C3 & $\mathrm{C} 4$ & \\
\hline \multicolumn{9}{|c|}{ Signal: $\tilde{g} \rightarrow t b \ell$ (scenario- 1$)$} \\
\hline$m_{\tilde{g}}=1.0$ & $370 \mathrm{fb}$ & {$[63]$} & $5 \times 10^{4}$ & 7704 & 7427 & 2463 & 2412 & 17.85 \\
\hline$m_{\tilde{g}}=1.5$ & $19 \mathrm{fb}$ & {$[63]$} & $5 \times 10^{4}$ & 6971 & 6846 & 2185 & 2184 & 0.83 \\
\hline$m_{\tilde{g}}=2.0$ & $1.56 \mathrm{fb}$ & {$[63]$} & $5 \times 10^{4}$ & 6218 & 6116 & 1846 & 1846 & 0.058 \\
\hline \multicolumn{9}{|c|}{ Backgrounds } \\
\hline$t \bar{t}+$ jets & $953.6 \mathrm{pb}$ & {$[64]$} & 11607567 & 4 & 4 & $<1$ & $<1$ & 0.0001 \\
\hline$t \bar{t} Z+$ jets & $1.121 \mathrm{pb}$ & {$[65]$} & 140734 & 2667 & 842 & 306 & 19 & 0.15 \\
\hline$t \bar{t} W+$ jets & $769 \mathrm{fb}$ & {$[66]$} & 169973 & 447 & 394 & 226 & 9 & 0.04 \\
\hline$Z Z W+$ jets & $44.3 \mathrm{fb}$ & {$[56]$} & 87650 & 272 & 52 & 4 & 1 & 0.0005 \\
\hline$W W Z+$ jets & $137.5 \mathrm{fb}$ & {$[56]$} & 65090 & 411 & 116 & 1 & $<1$ & - \\
\hline$W W W+\geq 2$ jets & $94.1 \mathrm{fb}$ & {$[56]$} & 26268 & 18 & 8 & $<1$ & $<1$ & - \\
\hline \multicolumn{9}{|l|}{ Total } \\
\hline Background & & & & & & & & 0.190 \\
\hline
\end{tabular}

Table 1. Event summary after individual selection cuts both for the MSSM benchmark points (in scenario-1) as well as the SM backgrounds for the multi-lepton analysis. The final cross-sections after all the selection cuts are shown in the last column. All the masses are in $\mathrm{TeV}$.
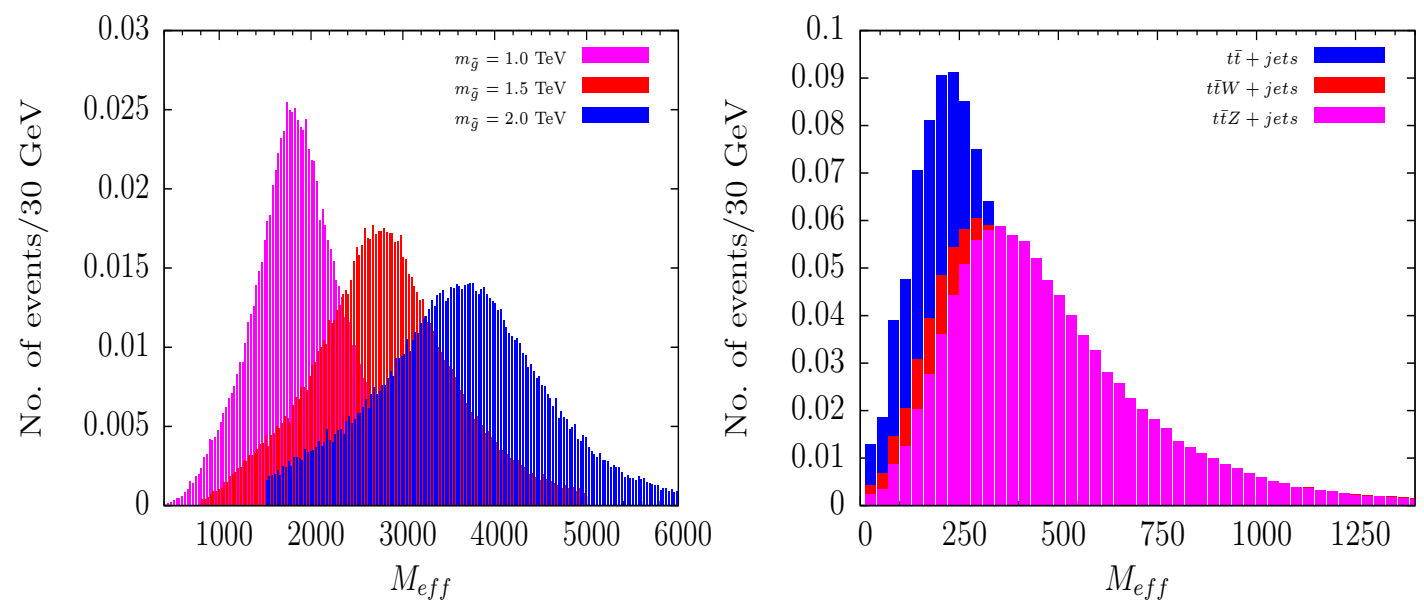

Figure 4. $M_{\text {eff }}$ distribution for three signal benchmark points as well as the dominant backgrounds. The total number of events has been normalized to unity.

each selection cut (described in section 3.1) are shown while the final column shows the cross-section after all the cuts have been imposed.

The dominant backgrounds in this case are $t \bar{t} Z+$ jets, $t \bar{t} W+$ jets and $t \bar{t}+$ jets. In case of $t \bar{t}+$ jets the semileptonic decay of the $B$-mesons (in addition to the two leptons from the $W$ 's) can contribute to the tri-lepton final state. Although the number of these events can be reduced to a good extent by requiring the isolation criteria as described in section 3.1, the enormous cross-section of the $t \bar{t}+$ jets process makes them significant background unless we impose hard lepton $p_{T}$ cuts. In fact, we have been able to reduce events from $t \bar{t}+$ jets 


\begin{tabular}{|c|c|c|c|c|c|c|c|c|}
\hline \multirow[b]{2}{*}{ Process } & \multirow[b]{2}{*}{$\begin{array}{c}\text { Production } \\
\text { cross-section }\end{array}$} & & & \multicolumn{4}{|c|}{ No. of events after the cut } & \multirow[b]{2}{*}{$\begin{array}{l}\text { Final cross- } \\
\text { section (fb) }\end{array}$} \\
\hline & & & $\begin{array}{c}\text { Simulated } \\
\text { events }\end{array}$ & $\mathrm{C} 1$ & $\mathrm{C} 2$ & C3 & $\mathrm{C} 4$ & \\
\hline \multicolumn{9}{|c|}{ Signal: $\widetilde{g} \rightarrow \widetilde{t}_{1} t, \widetilde{t}_{1} \rightarrow t \widetilde{\chi}_{1}^{0}, \widetilde{\chi}_{1}^{0} \rightarrow t b \ell($ scenario- 2$)$} \\
\hline$\left(m_{\tilde{g}}, m_{\tilde{t}}\right)=(1.0,0.8)$ & $370 \mathrm{fb}$ & {$[63]$} & $5 \times 10^{4}$ & 10609 & 8718 & 5663 & 5491 & 40.63 \\
\hline$\left(m_{\tilde{g}}, m_{\tilde{t}}\right)=(1.5,1.0)$ & $19 \mathrm{fb}$ & {$[63]$} & $5 \times 10^{4}$ & 10241 & 8794 & 6060 & 6058 & 2.3 \\
\hline$\left(m_{\tilde{g}}, m_{\tilde{t}}\right)=(2.0,1.0)$ & $1.56 \mathrm{fb}$ & {$[63]$} & $5 \times 10^{4}$ & 8706 & 7554 & 4823 & 4823 & 0.15 \\
\hline \multicolumn{9}{|c|}{ Backgrounds } \\
\hline$t \bar{t}+$ jets & $953.6 \mathrm{pb}$ & {$[64]$} & 11607567 & 316 & 300 & 87 & 10 & 0.82 \\
\hline$t \bar{t} Z+$ jets & $1.121 \mathrm{pb}$ & {$[65]$} & 140734 & 2870 & 887 & 350 & 21 & 0.167 \\
\hline$t \bar{t} W+$ jets & $769 \mathrm{fb}$ & {$[66]$} & 169973 & 594 & 512 & 272 & 15 & 0.067 \\
\hline$Z Z W+$ jets & $44.3 \mathrm{fb}$ & {$[56]$} & 87650 & 320 & 34 & 3 & 1 & 0.0005 \\
\hline$W W Z+$ jets & $137.5 \mathrm{fb}$ & {$[56]$} & 65090 & 467 & 162 & 2 & 1 & 0.002 \\
\hline$W W W+\geq 2$ jets & $94.1 \mathrm{fb}$ & {$[56]$} & 26268 & 34 & 23 & $<1$ & $<1$ & - \\
\hline \multicolumn{9}{|l|}{ Total } \\
\hline Background & & & & & & & & 1.05 \\
\hline
\end{tabular}

Table 2. Event summary after individual selection cuts both for the MSSM benchmark points (in scenario-2) as well as the SM backgrounds for the multi-lepton analysis. The final cross-sections after all the selection cuts are shown in the last column. All the masses are in TeV. We have taken the mass of $\widetilde{\chi}_{1}^{0}$ to be $300 \mathrm{GeV}$ in this case.

drastically after imposing $50 \mathrm{GeV}, 40 \mathrm{GeV}$ and $30 \mathrm{GeV}$ cuts respectively on first three hardest leptons (see table 1). Some events remain in scenario-2 (see table 2) where we lessen the $p_{T}$ cuts on leptons. The other dominant (the leading one in scenario-1) contribution comes from the $t \bar{t} Z+$ jets background where additional leptons can originate from the $Z$ boson decay. A $Z$ veto reduces this background substantially. The process $t \bar{t} W+$ jets also constitutes a background to our signal but its contribution is much less than $t \bar{t} Z+$ jets as can be seen from table 1 and 2 . We have also generated other potential backgrounds e.g., $Z Z W+$ jets, $W W Z+$ jets and $W W W+$ jets whose contributions are negligible compared to the previous ones when a $Z$ veto and the requirement of $2 b$-jets are also imposed. The use of the effective mass in the final step of the algorithm reduces backgrounds quite efficiently without affecting the signal almost at all. As the effective mass of an event for the signal is closely related to $2 m_{\widetilde{g}}$ the distribution peaks at very high values of the effective mass, see figure 4a where we show the effective mass distributions for three of our benchmark points with different choices of the gluino mass (in scenario-1). In figure $4 \mathrm{~b}$ we present the same distribution for the dominant backgrounds.

\subsection{Boosted top}

In this subsection we present our results using the techniques described in section 3.2. The summary of our findings is shown in table 3 where the same conventions as in table 1 and 2 have been used. In columns 4-7 the number of events after each selection cut (see section 3.2) are shown. The final column shows the final cross-section after all the selection cuts. 


\begin{tabular}{|c|c|c|c|c|c|c|c|c|}
\hline \multirow[b]{2}{*}{ Process } & \multirow{2}{*}{\multicolumn{2}{|c|}{$\begin{array}{l}\text { Production } \\
\text { cross-section }\end{array}$}} & \multirow[b]{2}{*}{$\begin{array}{c}\text { Simulated } \\
\text { Events }\end{array}$} & \multicolumn{4}{|c|}{ No. of events after the cut } & \multirow[b]{2}{*}{$\begin{array}{l}\text { Final cross- } \\
\text { section (fb) }\end{array}$} \\
\hline & & & & $\mathrm{C} 1$ & $\mathrm{C} 2$ & $\mathrm{C} 3$ & $\mathrm{C} 4$ & \\
\hline \multicolumn{9}{|c|}{ Signal: $\tilde{g} \rightarrow t b \ell$ (scenario- 1$)$} \\
\hline$m_{\tilde{g}}=1.0$ & $370 \mathrm{fb}$ & {$[63]$} & $5 \times 10^{4}$ & 7855 & 5257 & 2690 & 2681 & 19.83 \\
\hline$m_{\tilde{g}}=1.5$ & $19 \mathrm{fb}$ & {$[63]$} & $5 \times 10^{4}$ & 9007 & 6290 & 3044 & 3044 & 1.16 \\
\hline$m_{\tilde{g}}=2.0$ & $1.56 \mathrm{fb}$ & {$[63]$} & $5 \times 10^{4}$ & 9297 & 6521 & 2958 & 2958 & 0.09 \\
\hline \multicolumn{9}{|c|}{ Signal: $\widetilde{g} \rightarrow \widetilde{t}_{1} t, \widetilde{t}_{1} \rightarrow t \widetilde{\chi}_{1}^{0}, \widetilde{\chi}_{1}^{0} \rightarrow t b \ell($ scenario- 2$)$} \\
\hline$\left(m_{\tilde{g}}, m_{\tilde{t}}\right)=(1.0,0.8)$ & $370 \mathrm{fb}$ & {$[63]$} & $5 \times 10^{4}$ & 11982 & 3819 & 3023 & 2842 & 21.03 \\
\hline$\left(m_{\tilde{g}}, m_{\tilde{t}}\right)=(1.5,1.0)$ & $19 \mathrm{fb}$ & {$[63]$} & $5 \times 10^{4}$ & 15935 & 5438 & 4545 & 4534 & 1.72 \\
\hline$\left(m_{\tilde{g}}, m_{\tilde{t}}\right)=(2.0,1.0)$ & $1.56 \mathrm{fb}$ & {$[63]$} & $5 \times 10^{4}$ & 18130 & 5954 & 4973 & 4972 & 0.155 \\
\hline \multicolumn{9}{|c|}{ Backgrounds } \\
\hline$t \bar{t}+$ jets & $953.6 \mathrm{pb}$ & {$[64]$} & 31712564 & 424944 & 166 & 20 & 4 & 0.12 \\
\hline$t \bar{t} Z+$ jets & $1.121 \mathrm{pb}$ & {$[65]$} & 226110 & 9105 & 210 & 12 & 1 & 0.005 \\
\hline$t \bar{t} W+$ jets & $769 \mathrm{fb}$ & {$[66]$} & 276807 & 12105 & 164 & 10 & 1 & 0.003 \\
\hline$t \bar{t} h+$ jets & $700 \mathrm{fb}$ & {$[67]$} & 231064 & 10926 & 67 & 8 & 1 & 0.003 \\
\hline \multirow{2}{*}{$\begin{array}{l}\text { Total } \\
\text { Background }\end{array}$} & & & & & & & & \\
\hline & & & & & & & & 0.131 \\
\hline
\end{tabular}

Table 3. Event summary after individual selection cuts both for the MSSM benchmark points as well as the SM backgrounds for the boosted top analysis. The final cross-sections after all the selection cuts are shown in the final column. All the masses are in TeV. We have taken the mass of $\widetilde{\chi}_{1}^{0}$ to be $300 \mathrm{GeV}$ in scenario- 2 .

A comparison of columns 3 and 4, both for the signal and the backgrounds, clearly reveals the effectiveness of tagging an energetic top quark. In case of the $t \bar{t}+$ jets background the number of events get reduced by almost a factor of 75 while for the signal the loss is only by a factor of 5 . Such a large gain is due to the large average transverse energy (hence collimated jets) of the top quark in the signal compared to the background. For the background events the jets from the decay of a top quark are highly separated from each other and are not captured by a fat-jet which in turn reduces the tagging efficiency. The demand of two isolated leptons as well as two $b$-tagged jets also helps tame the background to a large extent. Note that these $b$-jets are far from the non- $W$ candidate of the tagged top quark (see section 3.2 for details). This criteria makes sure that the $b$-jets, most of the time, are not from the decay of the top quark which has been reconstructed.

In the last step of the analysis, the $M_{\text {eff }}$ cut brings down the background to a minuscule level keeping a handful of signal events. We have checked that the processes $t Z+$ jets, $t W+$ jets and $t h+$ jets do not contribute to our final number of background events.

\subsection{Comparison of the two analyses}

In table 4 we compute the signal significance obtained from the two analyses described above and compare them. We define the significance $\sigma$ as $\sigma=S / \sqrt{S+B}$ where $S$ and $B$ are the absolute number of signal and background events respectively for a particular 


\begin{tabular}{|c|c|c|c|c|}
\hline \multirow{2}{*}{$\begin{array}{c}\text { Gluino mass } \\
\text { (in GeV) }\end{array}$} & \multicolumn{2}{|c|}{ Scenario-1 } & \multicolumn{2}{c|}{ Scenario-2 } \\
\cline { 2 - 5 } & multi-lepton & boosted-top & multi-lepton & boosted-top \\
\hline 1000 & $29.71(42.02)$ & $31.38(44.38)$ & $44.5(62.9)$ & $32.32(45.71)$ \\
\hline 1500 & $5.81(8.22)$ & $7.21(10.20)$ & $8.88(12.56)$ & $8.93(12.64)$ \\
\hline 2000 & $0.82(1.16)$ & $1.35(1.91)$ & $0.96(1.37)$ & $2.04(2.89)$ \\
\hline
\end{tabular}

Table 4. The statistical significance of our signal for the two analysis strategies discussed in the text. The numbers outside (inside) the parentheses corresponds to an integrated luminosity of $50 \mathrm{fb}^{-1}\left(100 \mathrm{fb}^{-1}\right)$.

luminosity. At first we should mention that the raw signal cross-section reduces very fast as the gluino mass is increased; the cross-section drops to $1.56 \mathrm{fb}$ for a gluino mass of $2 \mathrm{TeV}$ from a healthy $370 \mathrm{fb}$ for a gluino mass of $1 \mathrm{TeV}$. This is the primary reason for the significance to drop dramatically with increasing $\widetilde{g}$ mass, as can be seen from table 4 .

We present the significance assuming two integrated luminosities, $50 \mathrm{fb}^{-1}$ and $100 \mathrm{fb}^{-1}$ (the numbers within the parentheses). It can be seen that for our first analysis strategy the significance is more than $8(12)$ for the $\mathcal{R}_{p}$ violating scenario-1 (scenario-2) with $\widetilde{g}$ mass of $1.5 \mathrm{TeV}$ and assuming $100 \mathrm{fb}^{-1}$ data set. Even at $50 \mathrm{fb}^{-1}$ integrated luminosity the significance $\sim 6(9)$ is achieved in scenario-1 (scenario-2). For a $2 \mathrm{TeV} \widetilde{g}$ mass the significance drops dramatically as can be seen from the last row of table 4 .

On the other hand, for the second analysis strategy the significance is about 7.2 (compared to about 5.8 in the multilepton case ) in the scenario- 1 for the $\widetilde{g}$ mass of $1.5 \mathrm{TeV}$ and $50 \mathrm{fb}^{-1}$ integrated luminosity. In scenario-2, however, both the analysis give similar results.

It is worth mentioning here that while calculating the significance we have not taken into account any systematic uncertainty which is very difficult to estimate in a reliable way. We believe that for the cases where the significance is large enough (say, $\geq 5$ ) the effect of including systematic uncertainties should not be large. For example, in the multilepton analysis in scenario-1 with gluino mass $1.5 \mathrm{TeV}$, adding a $30 \%$ systematic uncertainty on the background crosssection reduces the significance from 5.81 (8.22) to 5.65 (7.99) for $50 \mathrm{fb}^{-1}\left(100 \mathrm{fb}^{-1}\right)$ integrated luminosity.

\section{Conclusion}

Introduction of $\mathcal{R}_{p}$ violation in the MSSM lagrangian is a phenomenologically attractive way to evade strong bounds on the masses of SUSY particles obtained in the R-parity conserving scenario. This has fueled a significant amount of effort from both the experimentalists and theorists in investigating new signal topologies present in the R-parity violating case. However, there exists many more potentially interesting possibilities which still need to be covered. In this work, we have concentrated on two such topologies which exist in the presence of semileptonic R-parity violation with $\lambda_{133,233}^{\prime}$ couplings. We have considered gluino pair production with their subsequent decay to top quarks, leptons and $b$-jets. Two analysis strategies have been considered, one with the canonical multi-leptons and $b$-jets and the other one with more recent technique to reconstruct highly energetic 
top quarks. We performed a detailed simulation of the signal and all possible background processes to estimate and compare the effectiveness of these two procedures.

In order to present our results in a clear way we have chosen a few benchmark scenarios for both the topologies considered. Our results have been summarized in table 1, 2 and 3 and a comparison of the effectiveness of the two analyses procedures have been presented in table 4 . We observe that our second strategy which involves reconstructing a top quark from the final state was slightly more effective compared to the traditional multi-leptons + $b$-jets analysis. While in the multi-lepton with $b$-jets search the significance can reach up to as high as 5.8 (8.9) for a gluino mass of $1.5 \mathrm{TeV}$ in the $\mathcal{R}_{p}$ violating scenario-1 (scenario-2) with a $50 \mathrm{fb}^{-1}$ data set, the second analysis does somewhat better in scenario-1 (significance rises to 7.2$)$ and provides comparable sensitivity $(\sim 9)$ in the second scenario.

Note that, although we present our results in two simplified scenarios just for clear illustration of the procedures, our analysis can be applied to any other situations with similar final state. We would also like to mention that we have not considered any detector effects and pile-up contaminations which are rather difficult for us to simulate in a reliable way. However, we believe that our analysis can be taken as a guiding reference for more detailed and realistic analysis on real data by our experimental colleagues.

\section{Acknowledgments}

The research leading to these results has received funding from the European Research Council under the European Union's Seventh Framework Programme (FP/2007-2013) / ERC Grant Agreement n.279972. SB and DG would like to thank the members of the High Energy Theory Group at the Sapienza University of Rome for useful discussions. SN likes to acknowledge the computing facility at the Regional Centre for Accelerator-based Particle Physics (RECAPP), Harish-Chandra Research Institute. SN is also thankful to University of Helsinki and Helsinki Institute of Physics for the hospitality where the final part of the project has been carried out.

Open Access. This article is distributed under the terms of the Creative Commons Attribution License (CC-BY 4.0), which permits any use, distribution and reproduction in any medium, provided the original author(s) and source are credited.

\section{References}

[1] ATLAS collaboration, Observation of a new particle in the search for the standard model Higgs boson with the ATLAS detector at the LHC, Phys. Lett. B 716 (2012) 1 [arXiv: 1207.7214] [INSPIRE].

[2] CMS collaboration, Observation of a new boson at a mass of $125 \mathrm{GeV}$ with the CMS experiment at the LHC, Phys. Lett. B $\mathbf{7 1 6}$ (2012) 30 [arXiv:1207.7235] [INSPIRE].

[3] J.D. Lykken, Introduction to supersymmetry, hep-th/9612114 [INSPIRE].

[4] H.P. Nilles, Supersymmetry, supergravity and particle physics, Phys. Rept. 110 (1984) 1 [INSPIRE]. 
[5] S.P. Martin, A supersymmetry primer, hep-ph/9709356 [INSPIRE].

[6] H.E. Haber and G.L. Kane, The search for supersymmetry: probing physics beyond the standard model, Phys. Rept. 117 (1985) 75 [INSPIRE].

[7] ATLAS collaboration, Search for new phenomena in final states with large jet multiplicities and missing transverse momentum at $\sqrt{s}=8 \mathrm{TeV}$ proton-proton collisions using the ATLAS experiment, JHEP 10 (2013) 130 [arXiv:1308.1841] [INSPIRE].

[8] ATLAS collaboration, Search for strong production of supersymmetric particles in final states with missing transverse momentum and at least three b-jets using $20.1 \mathrm{fb}^{-1}$ of $\mathrm{pp}$ collisions at $\sqrt{s}=8 \mathrm{TeV}$ with the ATLAS detector, ATLAS-CONF-2013-061 (2013).

[9] B. Bhattacherjee, A. Dighe, D. Ghosh and S. Raychaudhuri, Do new data on $\left[B^{+} \rightarrow \tau^{+} \nu_{\tau}\right]$ decays point to an early discovery of supersymmetry at the LHC?, Phys. Rev. D 83 (2011) 094026 [arXiv: 1012.1052] [INSPIRE].

[10] A. Arbey, M. Battaglia, A. Djouadi, F. Mahmoudi and J. Quevillon, Implications of a 125 GeV Higgs for supersymmetric models, Phys. Lett. B 708 (2012) 162 [arXiv:1112.3028] [INSPIRE].

[11] D. Ghosh, M. Guchait, S. Raychaudhuri and D. Sengupta, How constrained is the cMSSM?, Phys. Rev. D 86 (2012) 055007 [arXiv: 1205.2283] [InSPIRE].

[12] A. Dighe, D. Ghosh, K.M. Patel and S. Raychaudhuri, Testing times for supersymmetry: looking under the lamp post, Int. J. Mod. Phys. A 28 (2013) 1350134 [arXiv:1303.0721] [INSPIRE].

[13] O. Buchmueller et al., The CMSSM and NUHM1 after LHC Run 1, arXiv:1312.5250 [INSPIRE].

[14] ATLAS collaboration, ATLAS SUSY 2013 stop summary, available online

[15] CMS collaboration, CMS SUSY 2013 stop summary, available online.

[16] D. Ghosh and D. Sengupta, Searching the sbottom in the four lepton channel at the LHC, Eur. Phys. J. C 73 (2013) 2342 [arXiv:1209.4310] [InSPIRE].

[17] A. Chakraborty, D.K. Ghosh, D. Ghosh and D. Sengupta, Stop and sbottom search using dileptonic $M_{T 2}$ variable and boosted top technique at the LHC, JHEP 10 (2013) 122 [arXiv: 1303.5776] [INSPIRE].

[18] D. Ghosh, Boosted di-boson from a mixed heavy stop, Phys. Rev. D 88 (2013) 115013 [arXiv: 1308.0320] [INSPIRE].

[19] G. Bélanger, D. Ghosh, R. Godbole, M. Guchait and D. Sengupta, Probing the flavor violating scalar top quark signal at the LHC, Phys. Rev. D 89 (2014) 015003 [arXiv: 1308.6484] [INSPIRE].

[20] J.S. Kim, M. Hanussek and M. Drees, Light stop phenomenology, PoS ICHEP2012 (2013) 112 [arXiv: 1304.7559] [INSPIRE].

[21] M.R. Buckley and D. Hooper, Are there hints of light stops in recent Higgs search results?, Phys. Rev. D 86 (2012) 075008 [arXiv:1207.1445] [InSPIRE].

[22] M. Carena, S. Gori, N.R. Shah, C.E.M. Wagner and L.-T. Wang, Light stops, light staus and the $125 \mathrm{GeV}$ Higgs, JHEP 08 (2013) 087 [arXiv: 1303.4414] [INSPIRE].

[23] G. Larsen, Y. Nomura and H.L.L. Roberts, Supersymmetry with light stops, JHEP 06 (2012) 032 [arXiv:1202.6339] [INSPIRE]. 
[24] X.-J. Bi, Q.-S. Yan and P.-F. Yin, Probing light stop pairs at the LHC, Phys. Rev. D 85 (2012) 035005 [arXiv:1111.2250] [InSPIRE].

[25] A. Datta and B. Mukhopadhyaya, Are messages of R-parity violating supersymmetry hidden within top quark signals?, Phys. Rev. Lett. 85 (2000) 248 [hep-ph/0003174] [INSPIRE].

[26] W. Porod, D. Restrepo and J.W.F. Valle, Light stop: MSSM versus R-parity violation, hep-ph/0001033 [INSPIRE].

[27] Z. Han, A. Katz, M. Son and B. Tweedie, Boosting searches for natural SUSY with RPV via gluino cascades, Phys. Rev. D 87 (2013) 075003 [arXiv: 1211.4025] [INSPIRE].

[28] J.A. Evans and Y. Kats, LHC coverage of RPV MSSM with light stops, JHEP 04 (2013) 028 [arXiv: 1209.0764] [INSPIRE].

[29] CMS collaboration, Search for supersymmetry in hadronic final states with missing transverse energy using the variables $\alpha_{T}$ and b-quark multiplicity in pp collisions at $8 \mathrm{TeV}$, Eur. Phys. J. C 73 (2013) 2568 [arXiv: 1303.2985] [INSPIRE].

[30] M. Lisanti, P. Schuster, M. Strassler and N. Toro, Study of LHC searches for a lepton and many jets, JHEP 11 (2012) 081 [arXiv:1107.5055] [INSPIRE].

[31] A. Hook, E. Izaguirre, M. Lisanti and J.G. Wacker, High multiplicity searches at the LHC using jet masses, Phys. Rev. D 85 (2012) 055029 [arXiv: 1202.0558] [INSPIRE].

[32] T. Cohen, E. Izaguirre, M. Lisanti and H.K. Lou, Jet substructure by accident, JHEP 03 (2013) 161 [arXiv: 1212.1456] [InSPIRE].

[33] J. Berger, M. Perelstein, M. Saelim and P. Tanedo, The same-sign dilepton signature of RPV/MFV SUSY, JHEP 04 (2013) 077 [arXiv: 1302.2146] [INSPIRE].

[34] D. Duggan et al., Sensitivity of an upgraded LHC to R-parity violating signatures of the MSSM, arXiv:1308.3903 [INSPIRE].

[35] J.A. Evans, Y. Kats, D. Shih and M.J. Strassler, Toward full LHC coverage of natural supersymmetry, arXiv:1310.5758 [INSPIRE].

[36] CMS collaboration, Search for top squarks in R-parity-violating supersymmetry using three or more leptons and b-tagged jets, Phys. Rev. Lett. 111 (2013) 221801 [arXiv:1306.6643] [INSPIRE].

[37] CMS collaboration, Search for RPV SUSY in the four-lepton final state, CMS-PAS-SUS-13-010 (2013).

[38] ATLAS collaboration, Search for massive particles in multijet signatures with the ATLAS detector in $\sqrt{s}=8 \mathrm{TeV}$ pp collisions at the LHC, ATLAS-CONF-2013-091 (2013).

[39] ATLAS collaboration, Search for a heavy particle decaying into an electron and a muon with the ATLAS detector in $\sqrt{s}=7 \mathrm{TeV}$ pp collisions at the LHC,

Phys. Rev. Lett. 106 (2011) 251801 [arXiv:1103.5559] [INSPIRE].

[40] ATLAS collaboration, Search for supersymmetry in events with four or more leptons in $13 \mathrm{fb}^{-1}$ pp collisions at $\sqrt{\mathrm{s}}=8 \mathrm{TeV}$ with the ATLAS detector, ATLAS-CONF-2012-153 (2012).

[41] ATLAS collaboration, Search for first generation scalar leptoquarks in pp collisions at $\sqrt{s}=7 \mathrm{TeV}$ with the ATLAS detector, Phys. Lett. B 709 (2012) 158 [Erratum ibid. 711 (2012) 442-455] [arXiv:1112.4828] [INSPIRE]. 
[42] ATLAS collaboration, Search for second generation scalar leptoquarks in pp collisions at $\sqrt{s}=7 \mathrm{TeV}$ with the ATLAS detector, Eur. Phys. J. C 72 (2012) 2151 [arXiv:1203.3172] [INSPIRE].

[43] CMS collaboration, Search for pair production of first- and second-generation scalar leptoquarks in pp collisions at $\sqrt{s}=7$ TeV, Phys. Rev. D 86 (2012) 052013 [arXiv:1207.5406] [INSPIRE].

[44] CMS collaboration, Search for pair production of third-generation leptoquarks and top squarks in pp collisions at $\sqrt{s}=7$ TeV, Phys. Rev. Lett. 110 (2013) 081801 [arXiv:1210.5629] [INSPIRE].

[45] ATLAS collaboration, Search for third generation scalar leptoquarks in pp collisions at $\sqrt{s}=7 \mathrm{TeV}$ with the ATLAS detector, JHEP 06 (2013) 033 [arXiv:1303.0526] [inSPIRE].

[46] H.K. Dreiner, An introduction to explicit R-parity violation, hep-ph/9707435 [INSPIRE].

[47] C. Schwanenberger, A review of searches for $R$ parity violating SUSY, Eur. Phys. J. C 33 (2004) S752 [hep-ex/0403013] [InSPIRE].

[48] R. Barbier et al., R-parity violating supersymmetry, Phys. Rept. 420 (2005) 1 [hep-ph/0406039] [INSPIRE].

[49] G. Bhattacharyya, R-parity violating supersymmetric Yukawa couplings: a minireview, Nucl. Phys. Proc. Suppl. 52A (1997) 83 [hep-ph/9608415] [INSPIRE].

[50] R.M. Godbole, P. Roy and X. Tata, Tau signals of R-parity breaking at LEP-200, Nucl. Phys. B 401 (1993) 67 [hep-ph/9209251] [InSPIRE].

[51] K. Agashe and M. Graesser, R-parity violation in flavor changing neutral current processes and top quark decays, Phys. Rev. D 54 (1996) 4445 [hep-ph/9510439] [INSPIRE].

[52] T. Takeuchi, O. Lebedev and W. Loinaz, Constraints on R-parity violation from precision electroweak measurements, hep-ph/0009180 [INSPIRE].

[53] CMS collaboration, Search for new physics in events with same-sign dileptons and $b$ jets in pp collisions at $\sqrt{s}=8 \mathrm{TeV}$, JHEP 03 (2013) 037 [Erratum ibid. 1307 (2013) 041] [arXiv:1212.6194] [INSPIRE].

[54] ATLAS collaboration, Searches for direct scalar top pair production in final states with two leptons using the stransverse mass variable and a multivariate analysis technique in $\sqrt{s}=8$ TeV pp collisions using 20.3 $\mathrm{fb}^{-1}$ of ATLAS data, ATLAS-CONF-2013-065 (2013).

[55] T. Sjöstrand, S. Mrenna and P.Z. Skands, PYTHIA 6.4 physics and manual, JHEP 05 (2006) 026 [hep-ph/0603175] [INSPIRE].

[56] M.L. Mangano, M. Moretti, F. Piccinini, R. Pittau and A.D. Polosa, ALPGEN, a generator for hard multiparton processes in hadronic collisions, JHEP 07 (2003) 001 [hep-ph/0206293] [INSPIRE].

[57] S. Hoeche et al., Matching parton showers and matrix elements, hep-ph/0602031 [INSPIRE].

[58] J. Pumplin et al., New generation of parton distributions with uncertainties from global QCD analysis, JHEP 07 (2002) 012 [hep-ph/0201195] [INSPIRE].

[59] D.E. Kaplan, K. Rehermann, M.D. Schwartz and B. Tweedie, Top tagging: a method for identifying boosted hadronically decaying top quarks, Phys. Rev. Lett. 101 (2008) 142001 [arXiv:0806.0848] [INSPIRE]. 
[60] M. Cacciari and G.P. Salam, Dispelling the $N^{3}$ myth for the $k_{t}$ jet-finder, Phys. Lett. B 641 (2006) 57 [hep-ph/0512210] [INSPIRE].

[61] M. Cacciari, G.P. Salam and G. Soyez, FastJet user manual, Eur. Phys. J. C 72 (2012) 1896 [arXiv:1111.6097] [INSPIRE].

[62] Y.L. Dokshitzer, G.D. Leder, S. Moretti and B.R. Webber, Better jet clustering algorithms, JHEP 08 (1997) 001 [hep-ph/9707323] [INSPIRE].

[63] W. Beenakker, R. Hopker and M. Spira, PROSPINO: a program for the production of supersymmetric particles in next-to-leading order QCD, hep-ph/9611232 [INSPIRE].

[64] M. Czakon, P. Fiedler and A. Mitov, Total top-quark pair-production cross section at hadron colliders through $O\left(\alpha_{S}^{4}\right)$, Phys. Rev. Lett. 110 (2013) 252004 [arXiv: 1303.6254] [InSPIRE].

[65] A. Kardos, Z. Trócsányi and C. Papadopoulos, Top quark pair production in association with a Z-boson at NLO accuracy, Phys. Rev. D 85 (2012) 054015 [arXiv:1111.0610] [INSPIRE].

[66] J.M. Campbell and R.K. Ellis, $t \bar{t} W^{+-}$production and decay at NLO, JHEP 07 (2012) 052 [arXiv: 1204.5678] [INSPIRE].

[67] S. Dawson, C. Jackson, L.H. Orr, L. Reina and D. Wackeroth, Associated Higgs production with top quarks at the large hadron collider: NLO QCD corrections,

Phys. Rev. D 68 (2003) 034022 [hep-ph/0305087] [INSPIRE]. 